\title{
An Engineered Distant Homolog of Pseudomonas syringae TTSS Effector From Physcomitrella patens Can Act as a Bacterial Virulence Factor
}

\author{
Marcin Piechocki', Fabian Giska', Grzegorz Koczyk ${ }^{2}$, Marcin Grynberg ${ }^{3}$ and \\ Magdalena Krzymowska ${ }^{1 *}$
}

'Institute of Biochemistry and Biophysics (PAS), Laboratory of Plant Pathogenesis, Warsaw, Poland, ${ }^{2}$ Institute of Plant Genetics (PAS), Department of Biometry and Bioinformatics, Poznań, Poland, ${ }^{3}$ Institute of Biochemistry and Biophysics (PAS), Department of Biophysics, Warsaw, Poland

\section{OPEN ACCESS}

Edited by:

Zhenyu Xie,

Hainan University, China

Reviewed by:

Christian Rueter,

Universitätsklinikum Münster

Germany

Luis G. C. Pacheco,

Universidade Federal da Bahia, Brazil

*Correspondence: Magdalena Krzymowska

krzyma@ibb.waw.pl

Specialty section: This article was submitted to

Evolutionary and Genomic

Microbiology,

a section of the journa

Frontiers in Microbiology

Received: 23 December 2017

Accepted: 04 May 2018

Published: 20 June 2018

Citation:

Piechocki M, Giska F, Koczyk G,

Grynberg $M$ and Krzymowska $M$

(2018) An Engineered Distant

Homolog of Pseudomonas syringae

TTSS Effector From Physcomitrella

patens Can Act as a Bacterial

Virulence Factor.

Front. Microbiol. 9:1060

doi: 10.3389/fmicb.2018.01060
Pseudomonas syringae pv. phaseolicola is the causative agent of halo blight in common bean (Phaseolus vulgaris). Similar to other pathogenic gram-negative bacteria, it secrets a set of type III effectors into host cells to subvert defense mechanisms. HopQ1 (for Hrp outer protein Q) is one of these type III effectors contributing to virulence of bacteria. Upon delivery into a plant cell, HopQ1 undergoes phosphorylation, binds host 14-3-3 proteins and suppresses defense-related signaling. Some plants however, evolved systems to recognize HopQ1 and respond to its presence and thus to prevent infection. HopQ1 shows homology to Nucleoside Hydrolases (NHs), but it contains a modified calcium binding motif not found in the canonical enzymes. CLuster ANalysis of Sequences (CLANS) revealed that HopQ1 and alike proteins make a distinct group of putative NHs located distantly from the classical enzymes. The HopQ1 - like protein (HLP) group comprises sequences from plant pathogenic bacteria, fungi, and lower plants. Our data suggest that the evolution of HopQ1 homologs in bacteria, fungi, and algae was independent. The location of moss HopQ1 homologs inside the fungal clade indicates a possibility of horizontal gene transfer (HGT) between those taxa. We identified a HLP in the moss Physcomitrella patens. Our experiments show that this protein (referred to as PpHLP) extended by a TTSS signal of HopQ1 promoted $P$. syringae growth in bean and was recognized by Nicotiana benthamiana immune system. Thus, despite the low sequence similarity to HopQ1 the engineered PpHLP acted as a bacterial virulence factor and displayed similar to HopQ1 virulence properties.

\footnotetext{
Keywords: HopQ1, Pseudomonas syringae, evolution, Physcomitrella patens, horizontal gene transfer, type three effector
}

\section{INTRODUCTION}

Plant pathogens have evolved several mechanisms to overcome host defense responses. One of these mechanisms relies upon the delivery of proteinaceous effectors into plant cells. Effectors are evolutionary shaped to target key elements of host immune signaling pathways. Plants in turn, evolved systems that sense some of these effectors and their perception initiates downstream 
signaling. It implicates that one effector may promote bacterial growth in one plant species whereas in other its presence may betray bacterial invasion to plant surveillance system. In most cases, however, plant receptors do not directly interact with their cognate effectors but rather sense specific modifications of the virulence targets introduced by the effectors (Cui et al., 2015).

Pseudomonas syringae is a widespread bacterium that can infect almost 200 plant species including important vegetable crops. Like many other gram-negative pathogenic bacteria, $P$. syringae injects type III effectors into host cells. The effectors contribute to disease development via manipulation of host defense system and physiology for the pathogen's benefit. As a whole species, $P$. syringae produces nearly 100 different effector families (Lindeberg et al., 2012; Büttner, 2016). Their number in particular strains is variable, with a minimal known effector repertoire restricted to nine proteins (Baltrus et al., 2011). The effectors shared between most strains are called core effectors and were possibly acquired before pathovars diversification (Rohmer et al., 2004). In addition to this process of vertical inheritance, the effector repertoires are shaped by horizontal gene transfer (Rohmer et al., 2004). Acquisition of a new effector may affect bacterial virulence properties in several ways. It may expand the range of host virulence targets, contribute to host shift or mask the function of another effector to avoid host recognition.

HopQ1 ( $\underline{H} r p / h r c$-dependent outer protein Q) is an effector recently acquired by $P$. syringae (Rohmer et al., 2004). The central region of HopQ1 shows overall homology to nucleoside hydrolases (nuc_hydro2). In contrast to the bona fide $\mathrm{NH}$ involved in purine/pyrimidine salvage pathways, the putative calcium-binding site within the predicted catalytic center of HopQ1 (DXXXDXDD) differs from the consensus sequence (DXDXXXDD). suggesting that the HopQ1 catalytic center may have evolved to process other substrates. Consistently, the purified recombinant HopQ1 neither cleaved nor bound standard substrates (Giska et al., 2013; Li et al., 2013) but it was shown by Hann et al. (2014) to hydrolyze in vitro the cytokinin precursor [iP-riboside $5^{\prime}$-monophosphate (iPRMP)].

Here we show that HopQ1 belongs to an old, widely spread protein family. To get insight into its evolution, we employed various approaches. Classical methods to infer horizontal gene transfer events are based on sequence composition or evolutionary history (Ravenhall et al., 2015). Due to high sequence divergence of HLPs (HopQ1 - like proteins) the composition based methods failed to identify HGT events. Topology of the phylogenetic tree did not allow us to exclude the hypothesis that HLPs belonging to various taxonomic groups evolved independently except for moss and fungal proteins that display strong grouping (60\%). Using a HopQ1like sequence from an unrelated organism, we performed experimental horizontal gene transfer. HLP from $P$. patens, engineered to mimic a bacterial TTSS effector displayed strong virulence properties in Phaseolus vulgaris but unexpectedly it triggered also defense response in Nicotiana benthamiana thereby determining $P$. syringae host range in a similar way to HopQ1. This suggests a functional equivalence of these two proteins.

\section{MATERIALS AND METHODS}

\section{Exploratory Analysis of HLP Subset Within the Nucleoside Hydrolase Superfamily}

Initially, sequences homologous to HopQ1 were found using jackhmmer online server ${ }^{1}$ (Finn et al., 2011). The search was performed against non-redundant (nr) database (up-to-date in January, 2015). Two iterations were performed, as additional rounds caused the inferred profile to lose similarity to the query protein (lowering the score of the corresponding hit). In order to reduce redundancy, all of the obtained hits were clustered by the CD-HIT online tool ${ }^{2}$ (Huang et al., 2010) with default options active (sequence identity cut-off at $90 \%$ ). The clustered sequences were checked manually and sequences truncated or lacking HopQ1-like or classical aspartic acid motif were removed.

The sequences from the database obtained previously were further supplemented by more HLPs from genome sequencing projects and all of the subjected to CLANS analysis ${ }^{3}$ (Frickey and Lupas, 2004) at the default options active. In particular, several fungal genome projects were searched via JGI/MycoCosm BLAST (E-value 1e-20, followed by a manual inspection) interface and draft moss genomes available at the time investigated for presence of $P$. patens-like HLP homologs (February, 2015). After that, main protein groups (clans) were manually annotated with taxonomic data available at NCBI/Taxonomy database.

\section{Phylogenetic Analyses of HLPs}

The amino acid sequences of HLPs and RihA (used as a rooting sequence) were aligned using MAFFT-L-INS-i (Katoh and Toh, 2008), manually adjusted to contain nucleoside hydrolase domain only (based on NCBI/CDD domain boundaries), then MAFFTL-INS-i tool was performed again. After that, the alignment was evaluated in T-COFFEE-TCS (Chang et al., 2014). The conserved core was kept for further analysis (all residues with TCS score equal or greater than 6).

The previously aligned sequences were put into Bayesian analysis in PhyloBayes version 1.5 (Lartillot et al., 2009) using implemented CAT and CAT-GTR models. Three independent chains were run for 40000 iterations and topological convergence criteria were assessed with PhyloBayes' bpcomp and tracecomp tools. Chains were sampled at every 5th iteration and first 5000 topologies were discarded as burnin. The best converged pair of chains was selected for consensus topology calculations and based on the support values and convergence criteria CAT-GTR result was chosen. Converged values of maximum and mean difference in supports were respectively: 0.12 and 0.01 , with discrepancies for all trace parameters below 0.1 and corresponding effective sample sizes all above 1000. The tree was rooted with classical nucleoside hydrolase RihA from Escherichia coli (GenBank Library Accession No. CQR80250.1)

\footnotetext{
${ }^{1}$ https://www.ebi.ac.uk/Tools/hmmer/search/jackhmmer

${ }^{2}$ http://weizhongli-lab.org/cdhit_suite/cgi-bin/index.cgi? cmd=cd-hit

${ }^{3}$ https://toolkit.tuebingen.mpg.de/\#/tools/clans
} 
as an outgroup and the visualization and annotation of the resulting tree was performed using MEGA7 (Kumar et al., 2016).

\section{P. syringae Strains and Inoculation}

Nicotiana benthamiana, tobacco (Nicotiana tabacum cv. 'Xanthi-nc'), and common bean (Phaseolus vulgaris 'Red Mexican') plants were grown in soil under controlled environmental conditions $\left(21^{\circ} \mathrm{C}, 16 \mathrm{~h}\right.$ of light, $8 \mathrm{~h}$ of dark), as described previously (Talarczyk et al., 2002). A sequence encoding PpHLP (GenBank Library Accession No. XP_001774397.1) was optimized for bacterial systems using online server OPTIMIZER ${ }^{4}$ (Puigbò et al., 2007) with 'guided random' option applied. Then, convenient restriction sites (absent in the modified sequence) were designed. The modified sequence with the restriction sites was synthesized in GENEART $^{5}$. A sequence encoding the TTSS signal of HopQ1 (Guttman et al., 2002) was added upstream to PpHLP, rihA, or mCherry sequences using primers containing appropriate restriction sites (Supplementary Table S1). The PCR product obtained and the restriction fragments containing P PHLP, rihA, or mCherry were triple ligated into a broad-hostrange pBBR1MCSXpTAC vector (Giska et al., 2013). The constructs were electroporated into appropriate $P$. syringae strains.

PtoDC3000 $\Delta 28$, a mutant strain of P. syringae pv. tomato DC3000 with 28 effector genes deleted (Kvitko et al., 2009) was used for virulence assays. The bacteria expressing HopQ1 or PpHLP were mixed at equal colony forming units (cfu) prior to inoculation $\left(10^{5} \mathrm{cfu} \mathrm{mL}^{-1}\right)$. Bacterial suspensions were infiltrated into leaves of 2-week-old bean 'Red Mexican' plants using a needleless hypodermic syringe. At selected time points, two $1-\mathrm{cm}$ diameter leaf disks were cut from infiltrated zones in each plant. Disks were superficially sterilized with $70 \%$ ethanol for $1 \mathrm{~min}$, rinsed with sterile water for $1 \mathrm{~min}$, and then ground in $300 \mathrm{~mL}$ of $10 \mathrm{mM} \mathrm{MgCl}_{2}$. Serial dilutions were plated onto LB agar plates. The bacteria were grown at $28^{\circ} \mathrm{C}$ and after 2 and 6 days replicated onto plates containing either kanamycin or gentamicin, which enabled strain differentiation and cfu counting. The competitive index (CI) was calculated as described previously (Macho et al., 2007). CI was defined as the ratio of the colonies corresponding to the strain carrying PpHLP to the strain expressing HopQ1 within the output samples, divided by the corresponding ratios in the input inocula. The results were compared statistically by Student's $t$-test, and differences were considered significant at $P<0.05$.

For avirulence tests, the plasmid encoding PpHLP was transformed into $P$. syringae pv. syringae $\mathrm{B} 728 \mathrm{a}$ strain. The bacterial cultures were adjusted to $10^{6} \mathrm{cfu} \mathrm{mL}^{-1}$ in MilliQ water and supplemented with Silwet L-77 (0.02\%). Five-weekold $N$. benthamiana plants were dip inoculated by inverting whole plants into bacterial suspensions and gently agitating for 30 s. Following inoculation, plants were placed immediately

\footnotetext{
${ }^{4}$ http://genomes.urv.es/OPTIMIZER/

${ }^{5} \mathrm{https} / / / \mathrm{www} \cdot$ thermofisher.com/pl/en/home/life-science/cloning/gene-synthesis/ geneart-gene-synthesis.html
}

under a plastic dome to maintain high humidity levels for $24 \mathrm{~h}$. Development of symptoms was assessed within 7 days.

\section{RESULTS}

\section{HLPs Are Widely Distributed and Make a Distinct Group Among Nucleoside Hydrolases}

It was inferred from the previous studies that HopQ1 has been recently acquired by $P$. syringae (Rohmer et al., 2004). In order to gain insight into its evolutionary history we analyzed the family of HopQ1 - like proteins (HLP). Jackhmmer analysis revealed over 3,600 sequences similar to HopQ1 containing non-canonical calcium binding site. Interestingly HLPs were not confined to plant pathogenic bacteria (e.g., P. syringae, Ralstonia spp., Xanthomonas spp., Acidovorax spp.) and fungi (e.g., Botryotinia fuckeliana, Marssonina brunnea, Sclerotinia sclerotiorum), but they were also found in algae (including Aureococcus anophagefferens, Thalassiosira sp., Emiliania huxleyi) and amongst several mosses (Physcomitrella patens, Pohlia nutans, Ceratodon purpureus).

To determine a position of HLPs in relation to other members of the nucleoside hydrolase superfamily we employed CLANS, a sequence similarity-based clustering method, which groups evolutionarily closest proteins together and then places them into a three-dimensional diagram (Frickey and Lupas, 2004). To this end, all the jackhmmer hits were clustered in CD-HIT, manually edited (Supplementary Table S2) and then subjected to CLANS. As shown in Figure 1 all the HLPs, containing the HopQ1-like calcium binding motif, grouped as, a distinct clan within the nucleoside hydrolase superfamily, that was distant from the classical core. This suggests that HLPs form a novel family of the proteins. Consistently, modeling of a few HLPs (Supplementary Figure S1) revealed that they share a common fold with RihA, a inosine/uridinepreferring nucleoside hydrolase from Escherichia coli, however their predicted structures are dissimilar to the classical nucleoside hydrolases.

\section{HLPs Evolved Independently At Least Three Times - An Unexpected Alliance of Mosses and Fungi}

To reconstruct evolutionary history of HLPs we built phylogenetic trees based on their $\mathrm{NH}$ domains. Amino acid sequences of HopQ1-like cluster homologs (Supplementary Table S2) were adjusted to comprise only the NH-like domain and the final alignment was performed (Supplementary Figure S2). On basis of a final, curated set of 56 sequences, a Bayesian phylogenetic tree was built. As shown in Figure 2, HLPs grouped in three large clades: bacterial, algal, and fungal. This suggests that in bacteria, algae, and fungi HLPs evolved largely independently. Bacterial clade consisted of the sequences coming from plant pathogens. HopQ1 formed the youngest branch of the tree. This is in line with the fact 


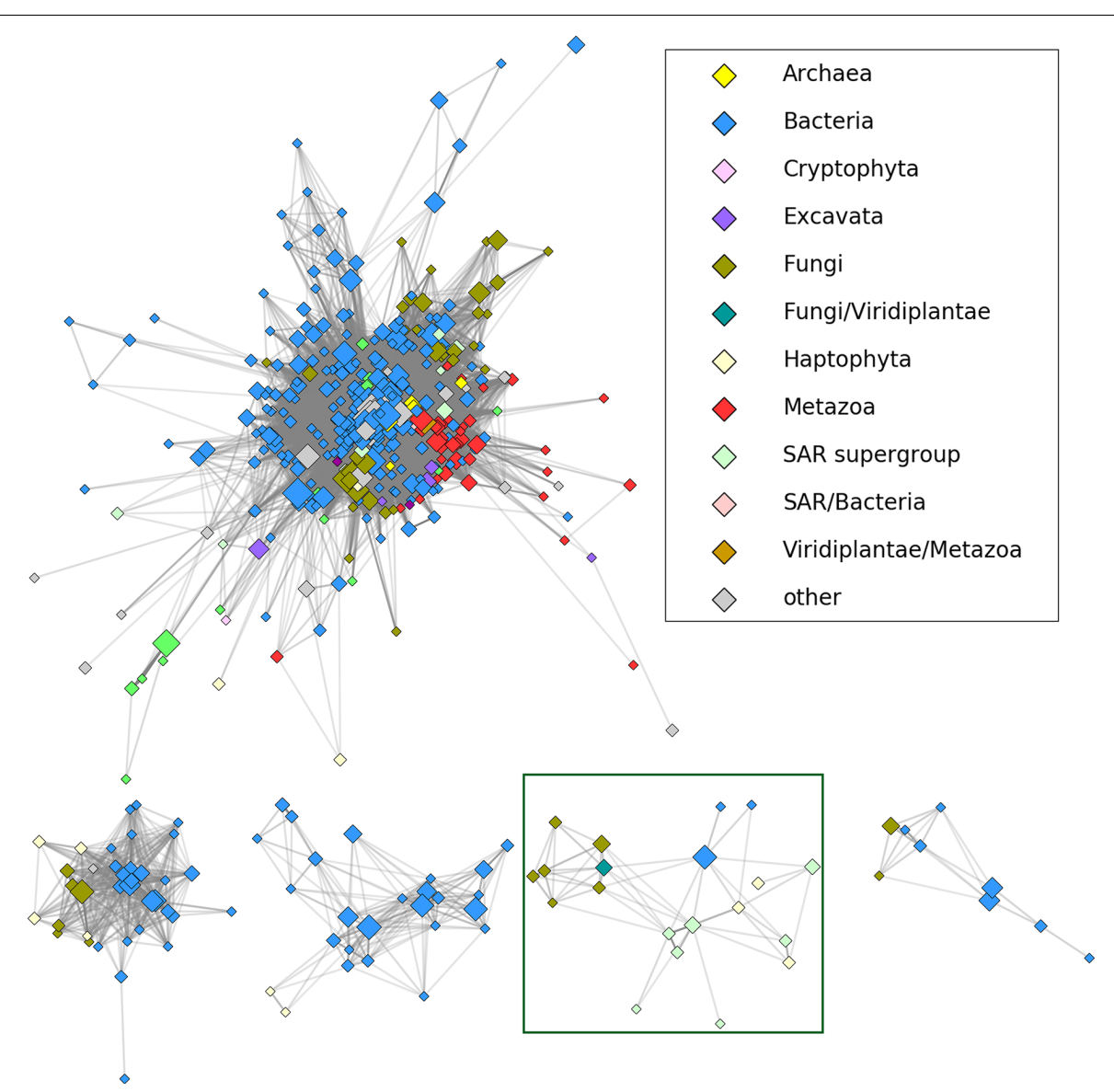

FIGURE 1 | The results of CLANS clustering of nucleoside hydrolase homologs at 1E-30 E-value threshold. HopQ1 amino acid sequence was used as a query to search for homologs using jackhmmer tool (https://www.ebi.ac.uk/Tools/hmmer/search/jackhmmer; sequence significance E-value - 0.01; hit significance E-value - 0.03). After two iterations, CD-HIT (http://weizhongli-lab.org/cdhit_suite/cgi-bin/index.cgi?cmd=cd-hit) (Huang et al., 2010) clustering at 90\% sequence identity cut-off and manual reduction of the number of hits, the remained sequences were analyzed by CLANS (https://toolkit.tuebingen.mpg.de/\#/tools/clans) program from the same toolkit. The CLANS program allows to join sequences with high homology and closest evolutionary connection into groups (clans). The five largest clans are depicted including HopQ1 homologs (HLPs). A reduced representation (sequences grouped at $70 \%$ percentage identity with CD-HIT) was used to visualize the connections in CytoScape (Shannon et al., 2003). Node color corresponds to taxonomic classification, node size is proportional to the number of sequences grouped (logarithmic scale). Shading of the edges corresponds to the best BLAST E-value (stronger similarities equal darker edges). The initial visualization was obtained in CytoScape using edge-weighted, spring-based layout and the final positions were manually adjusted for clarity. The HopQ1 clan is surrounded by a green line.

that HopQ1 acquisition by $P$. syringae occurred later than the recruitment of its homologs from other plant pathogens such as Xanthomonas spp. and Ralstonia spp. belonging to the core effectors (Rohmer et al., 2004). The sequences from heterokonts and haptophytes, including harmful brown tides causing agent $A$. anophagefferens, formed algal clade. Last clade was predominantly fungal, containing sequences from ascomycetes and basidiomycetes, including plant pathogens, saprotrophs, and mycorrhizal symbionts. Interestingly, moss clade was localized inside the fungal group. High bootstrap value supported the alliance between mosses and fungi in this context, with additional evidence pointing to nesting of the clade in between two groups of the fungal sequences (Figure 2). Thus the location of the moss clade indicated a possible horizontal gene transfer between those two distant groups.

\section{HLP From P. patens Promotes Faster Bacterial Growth in Common Bean Than HopQ1}

We asked further whether a HLP derived from an unrelated organism may perform a similar to HopQ1 biological function. To address this question we chose a HopQ1 homolog from moss $P$. patens, that is an established model organism with a well-annotated fully sequenced genome. The sequence encoding HLP from $P$. patens (PpHLP for $\underline{P}$. patens HopQ1-like protein) was optimized for expression in bacterial systems. To enable its delivery to plant cells by $P$. syringae, a sequence encoding the $\mathrm{N}$-terminal domain of HopQ1, comprising the TTSS secretion signal, was added upstream to PpHLP (Guttman et al., 2002). To assess virulence properties of the chimeric TTSS:PpHLP, we employed competitive index assay that compares growth of two 


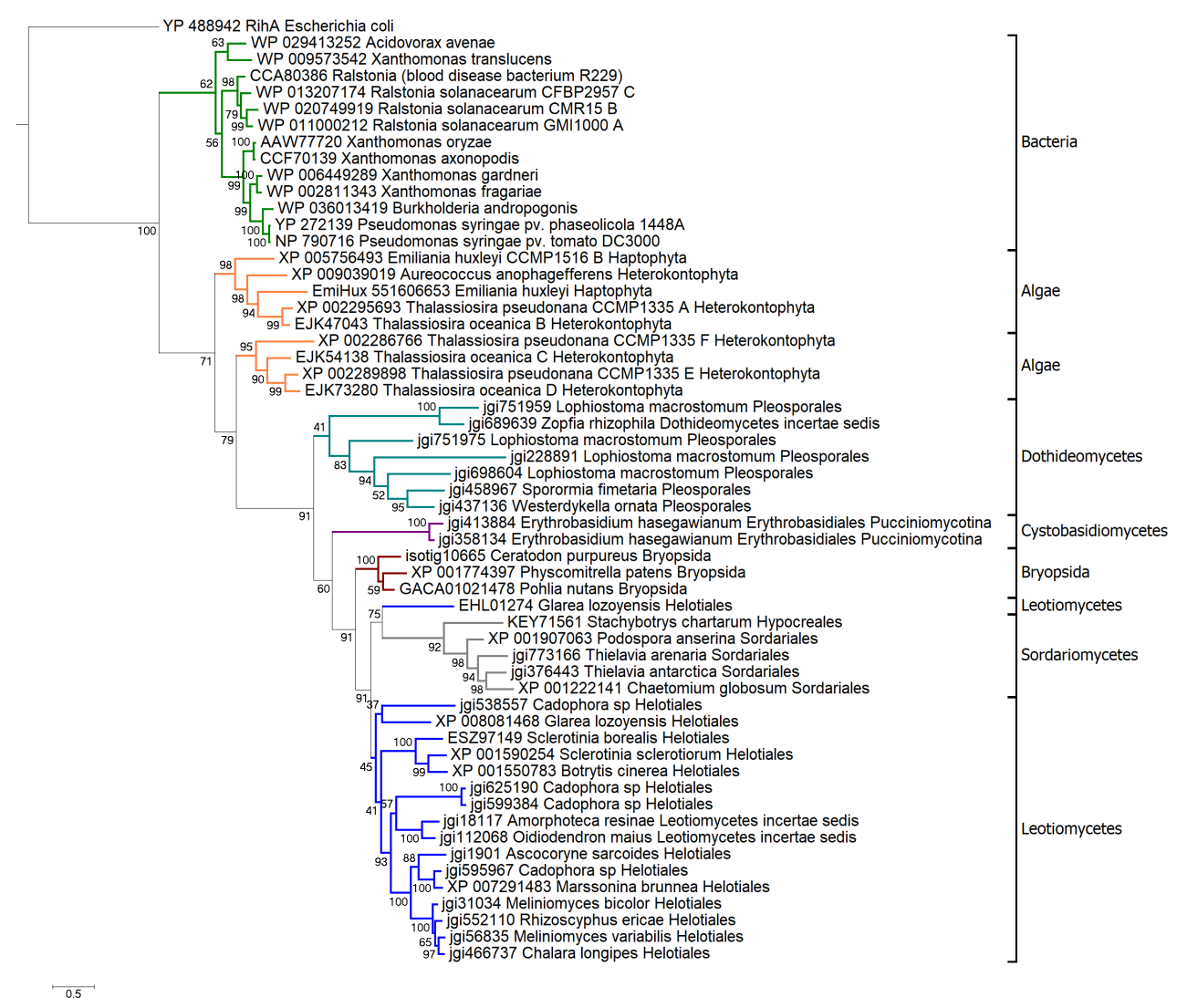

FIGURE 2 | Bayesian phylogenetic tree of HLPs. The tree was rooted with classical nucleoside hydrolase RihA from Escherichia coli. Branches representing main taxa were highlighted: bacteria in green, algae in orange, Dothideomycetes in teal, Sordariomycetes in gray, Cystobasidiomycetes in purple, mosses in brown, Leotiomycetes in navy. Each taxon is marked with black bracket. The analysis was carried out in PhyloBayes-MPI v. 1.5 (Lartillot et al., 2009). Three chains of 40000 iterations were run in parallel, two chains of best convergence were chosen for the consensus tree construction.

bacterial strains in mixed infection (Macho et al., 2007). To this end, the plasmid expressing TTSS:PpHLP was introduced into $P$. syringae pv. tomato DC3000 $\Delta 28 \mathrm{E}$, a strain deficient in 28 native effectors (Cunnac et al., 2011). Subsequently, bean leaves were infiltrated with a mixed inoculum (in a 1:1 ratio) of P. syringae strains expressing HopQ1 (Giska et al., 2013) and chimeric TTSS:PpHLP. At selected time points, bacteria were isolated from leaf tissue and plated onto solid media containing appropriate antibiotics. CI was calculated as the TTSS:PpHLP-toHopQ1 ratio within an output sample normalized for bacterial load. CI value of greater than 1 indicates the strain expressing TTSS:PpHLP proliferated significantly better than the one producing HopQ1, especially at the early phase of the infection (Figure 3). This indicates that TTSS:PpHLP strongly promoted virulence of $P$. syringae in bean.

\section{TTSS:PpHLP Is Recognized by N. benthamiana Immune System}

To check whether PpHLP engineered to resemble the bacterial virulence factor is recognized by plant immune system, TTSS:PpHLP expressing plasmid was transformed into P. syringae pv. syringae $\mathrm{B} 728 \mathrm{a}$, which highly virulent toward many species, including $N$. benthamiana (Vinatzer et al., 2006) and does not encode HopQ1. N. benthamiana plants were inoculated by dipping in bacterial suspensions, and then incubated for 7 days. Non-transformed bacteria, used as a control, caused severe disease symptoms in infected plants, which eventually died (Figure 4). As additional controls we prepared constructs encoding mCherry or RihA preceded by the TTSS of HopQ1 and we observed similar phenotypes for the plants infected with PsyB728a carrying them. As previously shown, expression of HopQ1 rendered the bacteria avirulent due to HopQ1 recognition by $N$. benthamiana immune system (Giska et al., 2013). Surprisingly, inoculation with the strain expressing TTSS:PpHLP did not cause any macroscopic disease symptoms - alike HopQ1 expressing strain. This indicates, that PpHLP, despite low sequence similarity to HopQ1 (25\% protein identity), triggers plant immune systems.

\section{DISCUSSION}

Previous bioinformatical studies showed, that proteins homologous to HopQ1 are widely spread among many species of plant pathogenic bacteria (Rohmer et al., 2004). In some genera, 


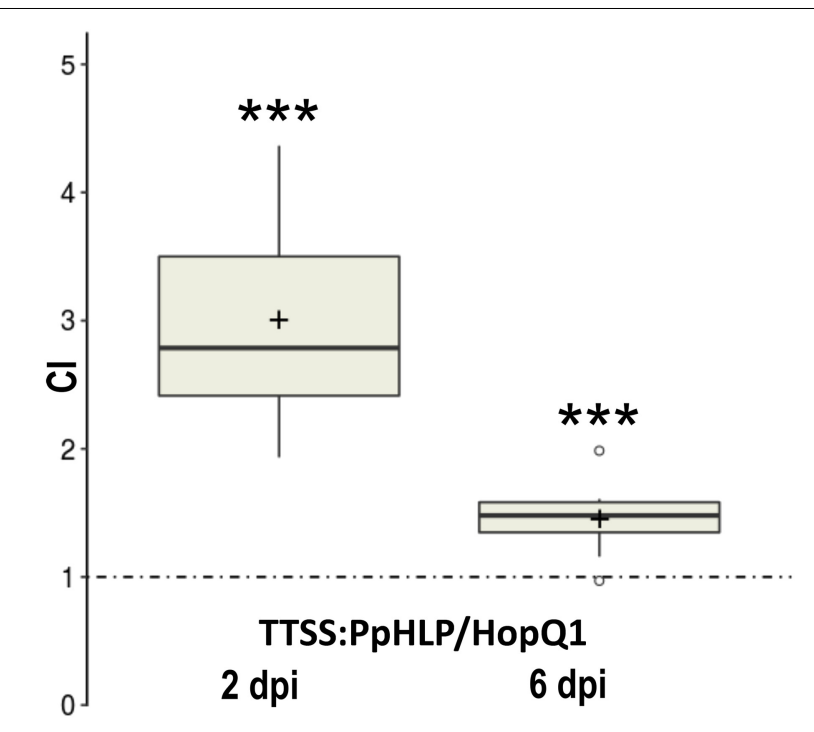

FIGURE 3 | Assessment of virulence properties of TTSS-PpHLP. Bean leaves were inoculated with Pseudomonas syringae pv. tomato DC3000 $\Delta 28 \mathrm{E}$ (approximately $10^{5} \mathrm{cfu} \mathrm{mL}^{-1}$ ) strains expressing HopQ1 or TTSS-PpHLP. Immediately prior to infiltration, bacteria were mixed in a 1:1 ratio. Two and 6 days post-inoculation (dpi), two leaf disks per plant were cut out from the infiltrated zones, ground in sterile $10 \mathrm{~mm} \mathrm{MgCl}_{2}$, diluted, and plated on LB medium. Bacterial strains were distinguished by a selectable marker. The $\mathrm{Cl}$ (competitive index) was calculated as the ratio of bacteria expressing TTSS-PpHLP to bacteria expressing wild-type HopQ1 isolated from plant leaf and normalized to the input titers of the bacteria. Asterisks indicate that the index is significantly different from 1 , as established using Student's $t$-test $(P<0.001)$. Pluses represent the means. The experiment was performed three times with similar results.

including Xanthomonas and Ralstonia, HopQ1 homologs belong to the core sets of effectors and are chromosomally encoded (Rohmer et al., 2004). In contrast, HopQ1 is present only in some $P$. syringae strains and it can be encoded either on the chromosome (e.g., P. syringae pv. tomato) or on a plasmid (e.g., $P$. syringae $\mathrm{pv}$. phaseolicola). Moreover, GC content, codon usage and presence of remnants of mobile elements in the neighborhood of hopQ1 sequence indicate a recent acquisition of this effector by P. syringae (Rohmer et al., 2004). This is consistent with HopQ1's position as the youngest branch of the bacterial clade in Bayesian tree of HLPs, supported by high bootstrap values (99) (Figure 2).

Our analyses showed that proteins homologous to HopQ1 can be found not only in plant pathogenic bacteria, but they also occur in fungi and lower plants such as algae and some moss species. Bayesian tree of HopQ1 homologs revealed at least three large clades consisting of bacterial, algal, and fungal proteins respectively. These groups are supported by high bootstrap values ( $>60$ ), which suggest that they evolved independently. What is interesting, the moss subclade is localized inside a bigger one, which contains fungal sequences. It is also well-supported by high bootstrap value (91). This may indicate a possible horizontal gene transfer between those two taxa.

Fungi interact with mosses by different means, and fungal-toplants gene transfers have been already reported (Richards et al., 2009). Various fungal species may infect mosses causing severe disease symptoms (Akita et al., 2011). Some, like Oidiodendron maius - a mycorrhizal symbiont of ericaceous plants - can live as endophytes and/or saprobes on Sphagnum peat moss (Thormann et al., 2002; Davey and Currah, 2006). Arbuscular mycorrhizal fungi also associate with mosses (Zhang and Guo, 2007).

When did some moss species obtain fungal (or fungal-like) HopQ1 homolog? The available evidence suggests that it might have occurred in early phase of land colonization by plants, before radiation of ascomycetes, at least 400 mya (millions of years ago). More than that, the presence of 'HopQ1' - encoding sequence in Erythrobasidium hasegawianum genome suggests that HGTs might have happened even earlier, prior to the diversification of ascomycetes and basidiomycetes, which occurred ca. 600 mya.

We have conducted an experimental HGT by introducing a sequence encoding HopQ1 homolog from moss $P$. patens (PpHLP) into $P$. syringae. This resulted in a significantly enhanced bacterial growth rate in bean, compared to hopQ1 carrying bacteria (Figure 3). Such scenario is usual for new, highly virulent pathogen species or strains, which did not coevolve with their actual host. Unexpectedly, despite the low sequence similarity to HopQ1 (25\% identity), expression of
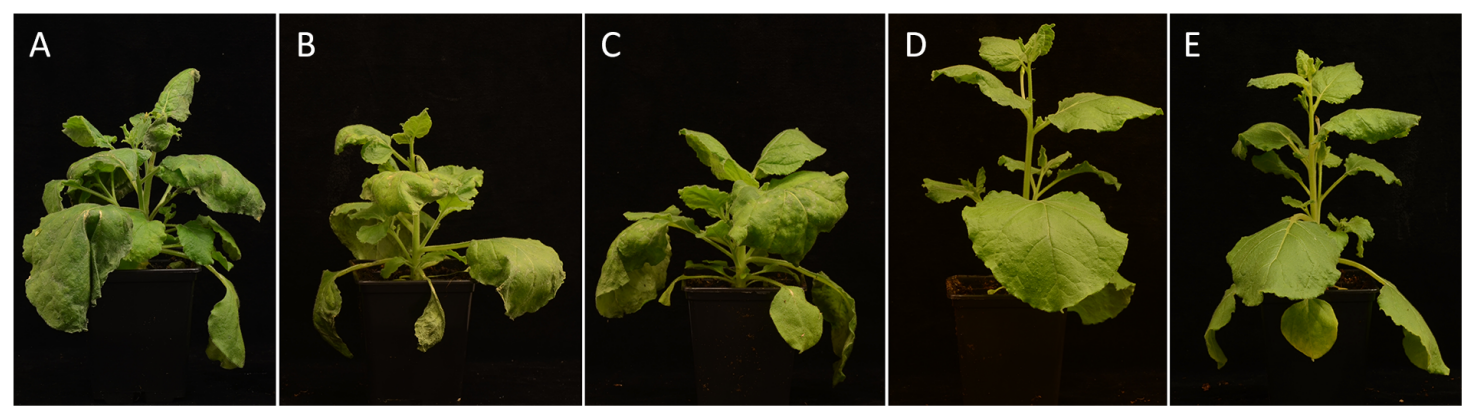

FIGURE 4 | PpHLP is recognized by plant immune system. Nicotiana benthamiana plants were inoculated with PsyB728a wild-type strain (A) or strains carrying pBBR1-MCS2 derivatives, which express TTSS-mCherry (B), TTSS-RihA (C), HopQ1 (D), or TTSS-PpHLP (E). Disease symptoms developed only in control plants, that is plants treated with the wild-type PsyB728a, PsyB728a carrying plasmids encoding TTSS-mCherry or TTSS-RihA. The photographs were taken 7 days post-inoculation. The experiment was performed twice, with similar results. 
TTSS:PpHLP induced defense response in N. benthamiana plants (Figure 4). This was possibly not due to the fragment of HopQ1 containing the secretion signal (TTSS) that had been added $\mathrm{N}$-terminally to PpHLP, since it was previously shown no to be recognized in Nicotiana spp. (Li et al., 2013). Moreover, previous studies indicated that no particular motif in HopQ1 sequence triggered defense response in $N$. tabacum leaves and it was inferred that the whole protein is required to induce immunity (Li et al., 2013; Hann et al., 2014). Together with the fact, that receptor Roq1 (Recognition of XopQ 1 ) of $N$. benthamiana mediates recognition of both HopQ1 and its close homolog from Xanthomonas spp. XopQ (Schultink et al., 2017), this would suggest the recognition of HLPs is indirect. In summary, introduction of the new virulence factor into $P$. syringae was advantageous to bacteria infecting the susceptible host, however the plant strategy to guard virulence targets turned to be successful in recognition of an unrelated factor by the resistant host.

The available phylogenetic evidence points to a monophyletic descent of the major clades (bacterial, two microalgal clades, and fungal/moss clade). Likewise an initial, exploratory analysis of base/codon compositions (principal components analysis in the sequence space) did not provide decisive argument regarding HopQ1 homolog origins in the aforementioned groups.

Concerning the HopQ1 homologs from moss, there is a clear evidence of grouping of the fungal and moss sequences (Figure 2). However, the nesting order does not provide sufficient support for horizontal transfer (the moss clade nested within the fungal sequences, with support of $60 \%$ in the final Bayesian consensus) rather than duplication (the moss clade located at

\section{REFERENCES}

Akita, M., Lehtonen, M. T., Koponen, H., Marttinen, E. M., and Valkonen, J. P. (2011). Infection of the Sunagoke moss panels with fungal pathogens hampers sustainable greening in urban environments. Sci. Total Environ. 409, 31663173. doi: 10.1016/j.scitotenv.2011.05.009

Baltrus, D. A., Nishimura, M. T., Romanchuk, A., Chang, J. H., Mukhtar, M. S., Cherkis, K., et al. (2011). Dynamic evolution of pathogenicity revealed by sequencing and comparative genomics of 19 Pseudomonas syringae isolates. PLoS Pathog. 7:e1002132. doi: 10.1371/journal.ppat.1002132

Büttner, D. (2016). Behind the lines-actions of bacterial type III effector proteins in plant cells. FEMS Microbiol. Rev. 40, 894-937. doi: 10.1093/femsre/ fuw026

Chang, J. M., Di Tommaso, P., and Notredame, C. (2014). TCS: a new multiple sequence alignment reliability measure to estimate alignment accuracy and improve phylogenetic tree reconstruction. Mol. Biol. Evol. 31, 1625-1637. doi: $10.1093 / \mathrm{molbev} / \mathrm{msu} 117$

Cui, H., Tsuda, K., and Parker, J. E. (2015). Effector-triggered immunity: from pathogen perception to robust defense. Annu. Rev. Plant Biol. 66, 487-511. doi: 10.1146/annurev-arplant-050213-040012

Cunnac, S., Chakravarthy, S., Kvitko, B. H., Russell, A. B., Martin, G. B., and Collmer, A. (2011). Genetic disassembly and combinatorial reassembly identify a minimal functional repertoire of type III effectors in Pseudomonas syringae. Proc. Natl. Acad. Sci. U.S.A. 108, 2975-2980. doi: 10.1073/pnas.10130 31108

Davey, M. L., and Currah, R. S. (2006). Interactions between mosses (Bryophyta) and fungi. Can. J. Bot. 84, 1509-1519. doi: 10.1139/b06-120

Finn, R. D., Clements, J., and Eddy, S. R. (2011). HMMER web server: interactive sequence similarity searching. Nucleic Acids Res. 39, W29-W37. doi: 10.1093/ nar/gkr367 the crown of the fungal clade). Following the principle of maximum parsimony we are thus forced to conclude that while both modeling and functional evidence from the "artificial" horizontal transfer corroborate a possibility of ancient transfers, the available genomic evidence was insufficient for a definite conclusion. In summary, for major clades from bacterial and eukaryotic microorganisms that are parallel, vertical descents from a common ancestor sequence remain a possibility.

\section{AUTHOR CONTRIBUTIONS}

FG, MP, GK, and MK conceived and designed the experiments. MP, FG, and GK performed the experiments. FG, MP, GK, $M G$, and $M K$ analyzed the data. MP and MK wrote the paper.

\section{FUNDING}

This work was supported by grants (Nos. 2013/11/B/NZ9/01970 and 2014/15/B/NZ3/04852 to MK and 2011/03/D/NZ2/01435 to GK) from the National Science Centre.

\section{SUPPLEMENTARY MATERIAL}

The Supplementary Material for this article can be found online at: https://www.frontiersin.org/articles/10.3389/fmicb. 2018.01060/full\#supplementary-material

Frickey, T., and Lupas, A. (2004). CLANS: a Java application for visualizing protein families based on pairwise similarity. Bioinformatics 20, 3702-3704. doi: 10 . 1093/bioinformatics/bth444

Giska, F., Lichocka, M., Piechocki, M., Dadlez, M., Schmelzer, E., Hennig, J., et al. (2013). Phosphorylation of HopQ1, a type III effector from Pseudomonas syringae, creates a binding site for host 14-3-3 proteins. Plant Physiol. 161, 2049-2061. doi: 10.1104/pp.112.209023

Guttman, D. S., Vinatzer, B. A., Sarkar, S. F., Ranall, M. V., Kettler, G., and Greenberg, J. T. (2002). A functional screen for the type III (Hrp) secretome of the plant pathogen Pseudomonas syringae. Science 295, 1722-1726. doi: 10. 1126/science.295.5560.1722

Hann, D. R., Domínguez-Ferreras, A., Motyka, V., Dobrev, P. I., Schornack, S., Jehle, A., et al. (2014). The Pseudomonas type III effector HopQ1 activates cytokinin signaling and interferes with plant innate immunity. New Phytol. 201, 585-598. doi: 10.1111/nph.12544

Huang, Y., Niu, B., Gao, Y., Fu, L., and Li, W. (2010). CD-HIT Suite: a web server for clustering and comparing biological sequences. Bioinformatics 26, 680-682. doi: 10.1093/bioinformatics/btq003

Kumar, S., Stecher, G., and Tamura, K. (2016). MEGA7: molecular evolutionary genetics analysis version 7.0 for bigger datasets. Mol. Biol. Evol. 33, 1870-1874. doi: 10.1093/molbev/msw054

Kvitko, B. H., Park, D. H., Velasquez, A. C., Wei, C. F., Russell, A. B., Martin, G. B., et al. (2009). Deletions in the repertoire of Pseudomonas syringae pv. tomato DC3000 type III secretion effector genes reveal functional overlap among effectors. PLoS Pathog. 5:e1000388. doi: 10.1371/journal.ppat.1000388

Lartillot, N., Lepage, T., and Blanquart, S. (2009). PhyloBayes 3: a Bayesian software package for phylogenetic reconstruction and molecular dating. Bioinformatics 25, 2286-2288. doi: 10.1093/bioinformatics/btp368

Li, W., Chiang, Y. H., and Coaker, G. (2013). The HopQ1 effector's nucleoside hydrolase-like domain is required for bacterial virulence in Arabidopsis and 
tomato, but not host recognition in tobacco. PLoS One 8:e59684. doi: 10.1371/ journal.pone.0059684

Lindeberg, M., Cunnac, S., and Collmer, A. (2012). Pseudomonas syringae type III effector repertoires: last words in endless arguments. Trends Microbiol. 20, 199-208. doi: 10.1016/j.tim.2012.01.003

Macho, A. P., Zumaquero, A., Ortiz-Martín, I., and Beuzón, C. R. (2007). Competitive index in mixed infections: a sensitive and accurate assay for the genetic analysis of Pseudomonas syringae-plant interactions. Mol. Plant Pathol. 8, 437-450. doi: 10.1111/j.1364-3703.2007.00404.x

Puigbò, P., Guzmán, E., Romeu, A., and Garcia-Vallvé, S. (2007). OPTIMIZER: a web server for optimizing the codon usage of DNA sequences. Nucleic Acids Res. 35, W126-W131. doi: 10.1093/nar/gkm219

Ravenhall, M., Škunca, N., Lassalle, F., and Dessimoz, C. (2015). Inferring horizontal gene transfer. PLoS Comput. Biol. 11:e1004095. doi: 10.1371/journal. pcbi. 1004095

Richards, T. A., Soanes, D. M., Foster, P. G., Leonard, G., Thornton, C. R., and Talbot, N. J. (2009). Phylogenomic analysis demonstrates a pattern of rare and ancient horizontal gene transfer between plants and fungi. Plant Cell 21, 1897-1911. doi: 10.1105/tpc.109.065805

Rohmer, L., Guttman, D. S., and Dangl, J. L. (2004). Diverse evolutionary mechanisms shape the type III effector virulence factor repertoire in the plant pathogen Pseudomonas syringae. Genetics 167, 1341-1360. doi: 10.1534/ genetics.103.019638

Schultink, A., Qi, T., Lee, A., Steinbrenner, A. D., and Staskawicz, B. (2017). Roq1 mediates recognition of the Xanthomonas and Pseudomonas effector proteins XopQ and HopQ1. Plant J. 92, 787-795. doi: 10.1111/tpj.13715

Shannon, P., Markiel, A., Ozier, O., Baliga, N. S., Wang, J. T., Ramage, D., et al. (2003). Cytoscape: a software environment for integrated models of biomolecular interaction networks. Genome Res. 13, 2498-2504. doi: 10.1101/ gr.1239303

Talarczyk, A., Krzymowska, M., Borucki, W., and Hennig, J. (2002). Effect of yeast CTAl gene expression on response of tobacco plants to tobacco mosaic virus infection. Plant Physiol. 129, 1032-1044. doi: 10.1104/pp.01 0960

Thormann, M. N., Currah, R. S., and Bayley, S. E. (2002). The relative ability of fungi from Sphagnum fuscum to decompose selected carbon substrates. Can. J. Microbiol. 48, 204-211. doi: 10.1139/w02-010

Vinatzer, B. A., Teitzel, G. M., Lee, M. W., Jelenska, J., Hotton, S., Fairfax, K., et al. (2006). The type III effector repertoire of Pseudomonas syringae pv. syringae B728a and its role in survival and disease on host and non-host plants. Mol. Microbiol. 62, 26-44. doi: 10.1111/j.1365-2958.2006.05350.x

Zhang, Y., and Guo, L. D. (2007). Arbuscular mycorrhizal structure and fungi associated with mosses. Mycorrhiza 17, 319-325. doi: 10.1007/s00572-0070107-8

Conflict of Interest Statement: The authors declare that the research was conducted in the absence of any commercial or financial relationships that could be construed as a potential conflict of interest.

Copyright (c) 2018 Piechocki, Giska, Koczyk, Grynberg and Krzymowska. This is an open-access article distributed under the terms of the Creative Commons Attribution License (CC BY). The use, distribution or reproduction in other forums is permitted, provided the original author(s) and the copyright owner are credited and that the original publication in this journal is cited, in accordance with accepted academic practice. No use, distribution or reproduction is permitted which does not comply with these terms. 